\title{
MedienPädagogik
}

Zeitschrift für Theorie und Praxis der Medienbildung

\section{OER - Auf dem Weg in eine selbstverschuldete digitale Unmündigkeit?}

Christopher Könitz

\begin{abstract}
Zusammenfassung
Spätestens seit der Debatte um die Vergütung von urheberrechtlich geschütztem Material nach UrhG \$52a, scheinen Open Educational Resources (OER) die Antwort auf proprietäre Verlagsangebote zu sein. Jedoch gibt es lizenzrechtliche, technische und begriffliche Unschärfen, die dazu führen, dass OER in eine selbstverschuldete digitale Unmündigkeit führen können. Dieser Beitrag liefert daher im Kern eine kritische Auseinandersetzung mit dem OER-Begriff aus einer bildungstheoretisch-medienpädagogischen Perspektive. Im ersten Teil werden die genannten Unschärfen näher beleuchtet und erste Lösungsansätze aufzeigt. Der Beitrag plädiert für eine Hinwendung zu einem starken Copyleft, welches derzeit mit den Creative Commons nicht möglich ist, da diese unter Umständen zu proprietären Materialen führen können. Die technische Perspektive richtet sich auf die verwendeten Dateiformate von OER, die häufig nicht frei und/oder editierbar sind. Die begriffliche Perspektive stellt heraus, dass der OER-Begriff ein negatives Konzept von Freiheit verfolgen und mit Blick auf die Medienpädagogik untertheoretisiert sind.

Im zweiten Teil werden daher durch das Medienkompetenzmodell nach Baacke und der Strukturalen Medienbildung nach Jörissen und Marotzki zwei mögliche medienpädagogische Anschlüsse geschaffen. Durch diese Anschlüsse werden die Momente der Medienkritik und der Reflexivität eingebracht. Damit wird der Fokus von einer Outputorientierung auf den Aufbau eines Orientierungswissens - und damit auf transformatorische Bildungsprozesse und den damit verbundenen medialen Artikulationen - verschoben.
\end{abstract}

OER - on it's way to a self-imposed digital immaturity?

\begin{abstract}
In the latest German debate on the remuneration of copyrighted material, Open Education Resources (OER) seem to be the answer to proprietary publishing offers. However, there are legal, technical and conceptual ambiguities in OER. These lead to a self-inflicted digital immaturity. This contribution is a critical examination of the OER concept from a media-pedagogical perspective. In the first part, the aforementioned ambiguities are examined in more detail. This article advocates a move towards a strong copyleft, which is currently not possible with the Creative Commons, as these may lead to proprietary materials. The technical perspective focusses on the file formats used by OER, which are
\end{abstract}

Könitz, Christopher. 2018. «OER - Auf dem Weg in eine selbstverschuldete digitale Unmündigkeit?». MedienPädagogik 32, (Oktober), 63-71. https://doi.org/10.21240/mpaed/32/2018.10.24.X. 
often not free and/or editable. The conceptual perspective shows that OER are based on a negative concept of freedom. Also, they are under-theorized in terms of media pedagogy. In the second part, two possible media pedagogical connections are illustrated. These are the media literacy model according to Baacke and the model of a structural media literacy according to Jörissen and Marotzki. Through these theoretical and practical approaches, the moments of media critique and reflexivity were combined with OER. This shifts the focus from an output oriented OER approach to transformative educational processes and medial articulations.

OER - Auf dem Weg in eine selbstverschuldete digitale Unmündigkeit? Mit der Debatte um die Einzelvergütung von urheberrechtlich geschütztem Material für Lehrzwecke, die die VG WORT angestossen hat, haben Open Educational Resources (OER) an Bedeutung gewonnen, da sie eine Antwort auf klassische proprietäre Verlagsangebote sind. Neben zahlreichen Tagungen und Barcamps, wie den OERBarcamps sowie dem OER-Festival, werden OER inzwischen auch durch Landes- und Bundesmittel unterstützt. Damit verbunden ist auch die Hervorhebung der positiven Aspekte dieser offenen Bildungsressourcen. Dabei lassen sich drei zentrale Argumente in der Debatte ausmachen. Erstens würden OER als Katalysator für die Digitalisierung der (Aus-)Bildung dienen, indem aus OER-Pools eigene Materialen und Kurse erstellt werden könnten. Zweitens minderten OER die digitale Kluft, indem Lernende sowie Bildungsinstitutionen einen freien Zugriff auf diese Materialen hätten (vgl. Ebner und Schön 2011, 2). Und drittens würden OER neue Formen des Lernens in Form von Open Educational Practices (OEP) eröffnen, da sie ideal für didaktische Szenarien wie das Remixen von Materialen seien (vgl. Mayrberger und Hofhues 2013, 59). Die Mindestanforderungen an OER werden häufig durch die sogenannten $5 R$ der Openess definiert:

- Retain - Das Recht Materialen zu kopieren, zu speichern und zu vervielfältigen.

- Reuse - Das Recht fremde Inhalte für eigene Zwecke weiterzuverwenden.

- Revise - Das Recht Inhalte zu überarbeiten bzw. anzupassen.

- Remix - Das Recht Inhalte zu mischen und neu zu arrangieren.

- Redistribute - Das Recht diese überarbeiteten oder originalen Versionen der Inhalte weiterzuverbreiten (vgl. Wiley 2004).

Dieser Fokus ist, auch mit Blick auf die OEP sehr funktional gefasst, da er vor allem Aspekte des Lernens und weiter gedacht von Bildung, nicht berücksichtigt. Dieser Beitrag liefert daher im Kern eine kritische Auseinandersetzung mit dem OER-Begriff aus einer bildungstheoretisch-medienpädagogischen Perspektive. Ausgangspunkt ist hierbei der von Kant formulierte Begriff der Aufklärung: 
«Aufklärung ist der Ausgang des Menschen aus seiner selbst verschuldeten Unmündigkeit. Unmündigkeit ist das Unvermögen, sich seines Verstandes ohne Anleitung eines anderen zu bedienen. Selbst verschuldet ist diese Unmündigkeit, wenn die Ursache derselben nicht am Mangel des Verstandes, sondern der Entschließung und des Muthes liegt, sich seiner ohne Leitung eines anderen zu bedienen. Sapere aude! Habe Muth, dich deines eigenen Verstandes zu bedienen! ist also der Wahlspruch der Aufklärung» (Kant 1784, 481).

Mit Blick auf dieses Zitat lautet meine These, dass die derzeitigen Konzeptionierungen von OER und OEP in eine selbstverschuldete digitale Unmündigkeit führen können. Daher werde ich im ersten Teil des Beitrags auf die derzeitigen kritischen Aspekte, die zu einer digitalen Unmündigkeit führen können, eingehen. Im zweiten Teil wird über das Medienkompetenzmodell nach Baacke (1998) und der Strukturalen Medienbildung (Jörissen und Marotzki 2009) ein bildungstheoretischer sowie -praktischer Anschluss geschaffen, der für OER und OEP fruchtbar gemacht werden kann.

\section{Die offenen Enden}

Waren es anfangs Sprachlabore oder Computerräume, sind es heutzutage Tabletklassen oder OER-Initiativen, die die Bildungsdiskussion treiben. Der gemeinsame Ausgangspunkt ist der gleiche: mittels neuer Medien, Materialien und Praktiken sollen Lernprozesse unterstützt und damit verbessert werden. Im Gegensatz zu den hardwarebasierten Innovationen, zeichnen sich OER zunächst durch eine freie Zugänglichkeit und den geringen Kostenfaktor für Lernende und Bildungsinstitutionen aus.

\section{Lizenzmodelle}

Bei genauerem Blick müssen beide Argumente jedoch in Teilen relativiert werden. Einerseits meint der freie Zugang meist, dass OER-Materialien unter einer freien Lizenz wie beispielsweise den Creative Commons (CC) stehen. Zusammen mit dem populären BY-Attribut, dass eine Namensnennung erforderlich macht, entsteht somit ein massgebliches rechtliches Hindernis für OER-Derivate (vgl. Kimpel 2017, 6f.). Insofern müssten OER auf ein starkes Copyleft setzen, um die praktische Wiederbenutzung zu erleichtern. Das Copyleft verfolgt die Idee, dass das Material in jeder Form bearbeitet werden darf unter der Bedingung, dass das editierte Format ebenfalls unter einem Copyleft weitergegeben wird. Somit wird verhindert, dass freie Formate in proprietäre Formate übergehen. Und hier würde auch der beliebte Hack der OER-Community, Materialen unter der in Deutschland derzeit unzulässigen $\mathrm{CCO}$ zu stellen, eben das Feld für proprietäre Derivate eröffnen. Insofern kann der Schlussfolgerung Kimpels widersprochen werden, man solle CC-Lizenzen nutzen, da diese weit verbreitet seien (vgl. Kimpel 2017, 9). Vielmehr kann dafür plädiert werden, dass die von Kimpel 
aufgeführte Kompatibilität der CC-Lizenz mit der General Public Licence genutzt werden sollte, um OER-Materialen mit einem starken Copyleft zu versehen.

\section{Dateiformate}

Neben der Frage nach freien Lizenzen ist auch die Frage nach den eigentlichen Dateiformaten ein kritisches Element von OER (vgl. Ebner und Schön 2011,3). Mit Blick auf den Diskurs um Open-Source-Software (OSS) und Free-Software, lassen sich wichtige Unterscheidungen für den OER-Diskurs ausmachen. OSS bedeutet, dass es neben einer freien Verfügbarkeit auch die Möglichkeit eines Einblicks in den Quellcode gibt. Das bedeutet jedoch nicht zwangsläufig, dass OSS in beliebiger Weise modifiziert werden darf. Insofern bildet die Free-Software-Bewegung einen kritischen Gegenpol zu OSS. Ausgangspunkt ist hierbei die Forderung, dass Software nur dann frei sei, wenn man sie beliebig ausführen, teilen und verändern dürfe (vgl. Free Software Foundation 2016). Grundlage dafür sind freie Werkzeuge und Formate. Mit Blick auf OER lässt sich feststellen, dass die meisten Materialen nicht so frei sind, wie es auf den ersten Blick scheint. Ein Beispiel hierfür ist das L3T. Freier Zugang bedeutet in diesem Beispiel ein Download als PDF-Datei, was ein proprietäres und nicht editierbares Dateiformat darstellt. Daneben gibt es die Varianten HTML-Seite und ePub. Diese sind freie Formate, können in diesem Beispiel aber ebenfalls nicht ohne weiteres Vorwissen editiert werden. Die HTML-Dokumente stehen nicht direkt zum Download bereit und das ePub-Format ist als Containerformat für Laien schwer nachvollziehbar. Insofern stärken diese Formate eine eher rezipierende Nutzung. Eine Variante im leicht zu editierenden Markdown-Format würde beispielsweise die Nachnutzung begünstigen.

Mit Blick auf andere OER lässt sich ein ähnliches Bild zeichnen. Das stellt ein wesentliches Problem in der OER-Debatte und -Praxis dar: proprietäre oder nicht editierbare Formate setzen sich in Form von Windows-PC-Pools, nicht herunterladbaren Youtube-Videos (vorzugsweise mit einer Standard-Youtube-Lizenz versehen) und iOS bzw. Android-Tablets in den OER und damit in OEP fort und erzeugen damit neue Abhängigkeiten sowie finanzielle Aufwände, die zugleich mediendidaktische Potenziale einschränken und hemmen.

\section{Kritik und Openess}

Neben diesen rechtlichen und technischen Unschärfen ist auch der Begriff der Openess im Kontext von OER und OEP unscharf konturiert. So stellt bspw. Markus Deimann zu dem Begriff der OEP zugespitzt fest: 
«Ein im Vergleich zu OER weniger bekannter Ansatz, mit dem als vorgeblich logische Fortführung von OER die nächste Stufe in einer Open Education Evolution proklamiert wird. In einer zumeist folkloristischen Rhetorik wird seit ungefähr zehn Jahren mehr oder weniger erfolglos versucht, offene pädagogische Modelle zu etablieren. Dabei kommt es zu einem eklatanten Missverhältnis zwischen dem selbstauferlegten Anspruch der Transformation von Bildung mit Hilfe des Katalysators OER und dem tatsächlich Erreichten. Noch 2016 ist es üblich, weniger von konkreten Maßnahmen zur Ausgestaltung von OEP zu sprechen als auf ideologisierende Weise an die Kraft offener Pädagogik zu appellieren und sich einer kritischen Auseinandersetzung zu verwehren» (Deimann 2016, 17).

Dieses Zitat impliziert zwei zentrale Kritiken: erstens die Kritik am zugrunde liegenden Begriff der Openess und zweitens eine Kritik an einer weitergehenden Auseinandersetzung mit bestehenden pädagogischen Diskursen.

Mit Blick auf die erste Kritik sollen die Überlegungen von Knox (2013) ins Feld geführt werden, die sich vor allem mit dem apriorischen Charakter von OER auseinandersetzen. Knox führt hierbei fünf Argumente, vor allem aus der Sicht von Bildungsinstitutionen, gegen den derzeitig vorherrschenden OER-Begriff an. Erstens komme es zu einer Untertheoretisierung von Freiheit und Offenheit. Im Rekurs auf positive (freedom to) und negative Freiheit (freedom from) nach Berlin (1969) konstatiert Knox, dass OER ein negativer Freiheitsbegriff immanent sei. Dies bedeutet nach Berlin, dass es weniger um eine persönliche Entfaltung, sondern vielmehr um die Festlegung der Grenzen dieser gehe (vgl. ebd. 1969, 15). Knox rekurriert anschliessend in seinem zweiten Argument auf das Paradoxon des Umgehens und des gleichzeitigen Forderns von institutionellen Strukturen. Hierbei spricht er einen Punkt an, der auch in der deutschsprachigen Diskussion anklingt. Auf der einen Seite sollten OER (und damit verbunden auch OEP) allen zugänglich sein. Insofern gibt es eine Tendenz (insb. in Form von Barcamps), diese von Institutionen wie Hochschulen zu lösen. Gleichzeitig wird eine institutionelle Neuverortung vorgenommen, wie beispielsweise durch die Informationsstelle für offene Bildungsmaterialen (OERinfo) des BMBF. Ein weiteres Beispiel ist die Forderung nach einem «deutschen Modell für OER» mit bestimmten Qualitätsmerkmalen und Beratungsangeboten (vgl. Mayrberger und Zawacki-Richter 2017, 78f.), dass dieses Paradoxon aufzeigt. In seinem dritten Argument konstatiert Knox, dass education zur Ware werden und die Verantwortung des Lernens stark bei den Lernenden verortet werden würde. Hierbei spricht sich Knox für die Notwendigkeit einer professionellen Begleitung aus, statt diese als Lernerleichterer zu verstehen (vgl. Knox 2013, 5). Das mit OER einhergehende Versprechen, dass nicht mehr Kontrollregime über Lerninhalte bestimmten würden, ist der vierte Punkt in Knox' Argumentation. Er geht in Anlehnung an Foucault davon aus, dass sich die Kontrollregime lediglich verschieben, jedoch nicht verschwinden würden (vgl. Knox 2013, 7f.). 
An diese ersten vier Argumente anschliessend, kommt Knox zu dem Schluss, dass OER letztlich nur dazu dienen würden, Humankapital auszubauen (vgl. Knox 2013, 8). Dieser kritischen Argumentation von Knox ist damit auch die Frage danach, was letztlich Bildung (als massgebliches pädagogisches Ziel) sei, inhärent. Im Folgenden wird daher, anschliessend an die oben erwähnte Kritik eines fehlenden pädagogisches Anschlusses im OER-Diskurs, die Argumentation dahingehend ausrichtet, inwiefern ein medienpädagogisches Modell, in Form einer positiv gedachten Freiheit (freedom to) für OER aussehen könnte, die den emanzipatorischen Gedanken im Sinne der Aufklärung verfolgt.

\section{Ein Schritt zurück: Medienkompetenz und OER}

Bisher wurde der Diskurs um OER zwar innerhalb der Mediendidaktik geführt, jedoch nicht ausreichend an bestehende medienpädagogische Diskurse angeschlossen. Dabei lassen sich hier durchaus gewinnbringende Anschlüsse herstellen, die zugleich eine Einordnung dieses Phänomens für die Medienpädagogik ermöglichen. Der erste Anschluss für OER und OEP erfolgt an das Medienkompetenzmodell nach Dieter Baacke (1997). Dieses umfasst vier Dimensionen. Medienkritik meint das analytische Erfassen gesellschaftlicher Prozesse, den reflexiven Umgang mit diesem Wissen und das Einflechten ethischer Aspekte darin. Mit dem Begriff der Medienkunde ist ein Wissen um Medien gemeint. Dieses Wissen differenziert Baacke in eine informative Dimension (Wissen über Medien(-systeme)) und in eine instrumentell-qualifikatorische Dimension (Allgemeine Fähigkeit sich in neue Medien einzuarbeiten). Die Dimension der Mediennutzung zielt daran anschliessend auf die Nutzung ab, die rezeptiv-anwendend oder interaktiv-anbietend sein kann. Den Bereich der Mediengestaltung unterteilt Baacke in einen innovativen und in einen kreativen Teil. Innovative Mediengestaltung zielt hier auf Weiterentwicklungen bestehender Medien und Medienformate ab, während Baacke in der kreativen Mediengestaltung die ästhetischen und grenzüberschreitenden Aspekte von Kommunikation unterstreicht (vgl. Baacke 1997, 98f.).

Mit Blick auf das oben vorgestellte 5R-Modell nach Wiley lassen sich durchaus Überschneidungen mit dem Medienkompetenzmodell nach Baacke ausmachen. Das Nutzen, Verbreiten und Verwahren kann der Dimension der Mediennutzung zugeschrieben werden. Das Verarbeiten und Vermischen kann der Dimension der Mediengestaltung zugeordnet werden. Die Dimension der Medienkunde könnte ein Wissen über rechtliche Aspekte wie freie Lizenzmodelle beinhalten, dass gerade in der deutschsprachigen Diskussion ein zentrales Thema ist. Das Moment der Medienkritik scheint im Kontext der 5R hingegen kein Äquivalent zu finden. Hierbei lässt sich also aus medienpädagogischer Sicht durchaus eine Leerstelle ausmachen. Dies kann begründet werden im bereits erwähnten funktionalen Charakter der 5R-Definition. Insofern kann und muss die Medienpädagogik ein sechstes $\mathrm{R}$ beitragen: Das 
der Reflexivität. Denn Reflexivität ist die Grundlage für Mündigkeit und damit auch für Bildung. Praktisch betrachtet könnte diese Reflexivität in einer kritischen Auseinandersetzung mit Materialien, die nicht nur Inhalte, sondern auch Formen und Transformationen zum Gegenstand macht, liegen.

«Hier setzt die ergänzende Erschließung kraft des Begriffs ,Bildung' ein, die darin bestände, daß die Unverfügbarkeit des Subjekts sich nach dessen eigenen generativen Ausdrucksmustern entfaltet, ohne durchweg immer pädagogisch und im pädagogischen Raum angeleitet sein zu müssen. Die Dimension von ,Erziehung' (im weitgefassten Sinn) und ,Bildung' sind in ,Medienkompetenz' einzudenken» (Baacke 1997, 100).

Mit dem Anschluss von OER an das Medienkompetenzmodell von Baacke zeigt sich, dass der durch Knox hervorgebrachten Kritik durchaus seitens der Medienpädagogik begegnet werden kann. Gerade weil der Medienkompetenzbegriff nicht nur institutionell und explizit als empanzipatives freedom to gedacht wird. Insofern soll der durch Baacke angerissene Bildungsbegriff in Form der Strukturalen Medienbildung im Kontext von OER im Folgenden vertiefen werden.

\section{Ein Schritt vor: Strukturale Medienbildung im Kontext von OER}

Die von Jörissen und Marotzki (2009) formulierte Strukturale Medienbildung bildet einen weiteren gewinnbringenden Anschlusspunkt für OER und OEP, da diese im Anschluss an bildungs- und modernisierungstheoretische Überlegungen fragt, inwiefern mediale Artikulationen zu einem Aufbau von Orientierungswissen führen können. Dieser Medienbildungsbegriff fokussiert hierbei nicht auf eine Outputorientierung, sondern wird als «Bezeichnung für transformatorische Prozesse (,Bildungsprozesse') im Horizont von Medialität» (Jörissen 2011, 87) begriffen. Insofern würde diese Perspektive OER und OEP weniger aus Ressource für die Stärkung eines Verfügungswissens sehen. Vielmehr wären OER und OEP eine Auseinandersetzung und ein Einlassen auf das Moment der Unbestimmtheit, die keinesfalls in Bestimmtheit enden muss, sondern auch Paradoxien und tentativen Suchbewegungen hervorbringen kann (vgl. Marotzki und Jörissen 2008, 56). Der Aufbau eines Orientierungswissens, dass zu einer Selbstbestimmung des Individuums führen würde (Mittelstrass 2002, 166), wäre daher der wesentliche Fokus einer reflexiven OEP, die sich in OER artikulieren würde.

Damit verbunden, würden sich zwei Handlungsfelder eröffnen. Erstens läge der didaktische Fokus auf der Auseinandersetzung mit der jeweiligen spezifischen Medialität von OER (bspw. in Formate, Formen, Möglichkeiten oder Handlungsprozessen). Diese so gedachte Auseinandersetzung mit OER, würden den Kern einer um Reflexion bzw. Medienkritik erweiterten offenen Lehr- und Lernpraxis bilden. Gleichsam könnten auch Digital Media Literacies (vgl. Jenkins 2010) bei allen Partizipierenden 
kreativ und tentativ weiterentwickelt werden. Hierfür ist das oben ausgeführte starke Copyleft essenziell. Die so ermöglichten medialen Artikulationen würden wiederum Bildungspotenziale eröffnen (vgl. Jörissen und Marotzki 2009, 39f.), die durch nonformale wie auch formale offene Bildungspraxen (vgl. Mayrberger und Hofhues 2013, 60f.) unterstützt werden könnten und letztlich zu einer digitalen Mündigkeit führen können. Damit verbunden wäre das zweite Handlungsfeld. Denn OER und OEP wären mit dem Anschluss an die Strukturale Medienbildung in theoretischer wie auch analytischer Hinsicht, stärker an eine bildungswissenschaftlich orientierte Medienpädagogik angebunden. Hierbei liegt ein ergiebiges Forschungsfeld, dass die Bedingungen von Bildungspotenzialen und -prozessen, die durch OER und OEP eröffnet werden könnten, in den Blick nehmen würde. Dies würde zugleich den Fokus von OER und OEP neu ausrichten. Nicht mehr die Frage nach der Qualität oder der inhaltlichen und juristischen Absicherung, sondern die Frage nach Emergenz, Unbestimmtheit und den kreativen Umgang damit stünden im Mittelpunkt.

\section{Fazit}

Mit Blick auf die Kriterien von OER in Form der $5 R$ lässt sich feststellen, dass derzeitige Konzeptionierungen von OER eher funktional gefasst werden. Zudem gibt es in der Frage nach den Lizenzmodellen sowie den Dateiformaten offene Fragen, die durchaus OEP und die damit verbundene Generierung von OER hemmen und zudem in eine digitale Unmündigkeit führen können. Es lässt sich daher konstatieren, dass der Argumentationsmodus von OER und OEP sich auf ein it just works fokussiert. Dies äussert sich auch im Auslassen von erziehungswissenschaftlichen und medienpädagogischen Anschlüssen. Dabei sind diese, wie das Medienkompetenzmodell nach Baacke und die Strukturale Medienbildung zeigen, durchaus eine fruchtbare Erweiterung für OER und OEP, da diese das Moment der Medienkritik bzw. der Reflexivität inkludieren. Die aus dieser Verschränkung hervorgehende gedachte und gelebte Mediendidaktik wäre dann eine solche, die eine durch Modernisierungsprozesse zunehmend paradoxe und unbestimmte Welt in den Blick nimmt und dabei hilft den Aufbau eines Orientierungswissens zu unterstützen.

\section{Literatur}

Baacke, Dieter. 1997. Medienpädagogik. Tübingen: Niemeyer.

Berlin, Isaiah. 1969. Two Concepts of Liberty. Zugriff am 30.1.2018. http://faculty.www.umb. edu/steven.levine/courses/Fall\%202015/What\%20is\%20Freedom\%20Writings/Berlin.pdf.

Deimann, Markus. 2016. "Open Education - die ewig Unvollendete». Synergie. Fachmagazin für Digitalisierung in der Lehre. 2. Ausgabe, 2016, S. 14-19. http://nbn-resolving.de/ urn:nbn:de:101:1-201612019830. 
Ebner, Martin, und Sandra Schön. 2011. «Offene Bildungsressourcen: Frei zugänglich und einsetzbar». In Handbuch E-Learning. Expertenwissen aus Wissenschaft und Praxis - Strategien, Instrumente, Fallstudien, hrsg. v. Karl Wilbers und Andreas Hohenstein. 39. Erg.-Lfg., 1-14. Köln: Deutscher Wirtschaftsdienst. http://www.scribd.com/doc/67768781/OffeneLernressourcen-Frei-zuganglich-und-einsetzbar.

Free Software Foundation. 2016. «Freie Software. Was ist das?». (Webseite). https://www.gnu. org/philosophy/free-sw.

Jenkins, Henry. 2010. «Multiculturalism, Appropriation, and the New Media Literacies: Remixing Moby Dick». In Mashup Cultures, hrsg. v. Stefan Sonvilla-Weiss, 98-199. Wien: Springer. https://doi.org/10.1007/978-3-7091-0096-7_7.

Jörissen, Benjamin. 2011. «>Medienbildung< - ein Konzept in heterogenen institutionellen Verwendungskontexten». In Medien \& Bildung, hrsg. v. Torsten Meyer, Wey-Han Tan, Christina Schwalbe, und Ralf Appelt, 83-91. Wiesbaden, VS Springer. https://doi.org/10.1007/9783-531-92082-5_6.

Jörissen, Benjamin, und Winfried Marotzki. 2009. Medienbildung - Eine Einführung. 1. Aufl., Stuttgart: UTB.

Kant, Imanuel. 1784. «Beantwortung der Frage: Was ist Aufklärung?». Berlinische Monatsschrift 2 (Dezember): 481-494.

Kimpel, Paul. 2017. «Und Wieder, und wieder, und wieder - Rechtsprobleme bei wiederholter Nutzung frei lizenzierter Inhalte». i.Right.Law-Anwälte für eine digitale Zukunft. Expertise im Auftrag der Zentralstelle für Lehren und Lernen im 21. Jahrhundert e.V. für OERcamp on Tour. http://www.oercamp.de/wp-content/uploads/2017/11/Klimpel-OER-Expertise-fuerOERcamp.pdf.

Knox, Jeremy. 2013. «Five Critiques of the Open Educational Resources Movement». Teaching in Higher Education 18 (8): 821-32. https://doi.org/10.1080/13562517.2013.774354.

Marotzki, Winfried, und Benjamin Jörissen. 2008. «Wissen, Artikulation und Biographie: theoretische Aspekte einer Strukturalen Medienbildung». In Pädagogische Medientheorie, hrsg. v. Johannes Fromme und Werner Sesink, 51-70. Wiesbaden: VS Verlag für Sozialwissenschaften. https://doi.org/10.1007/978-3-531-90971-4_4.

Mayrberger, Kerstin, und Sandra Hofhues. 2013. «Akademische Lehre braucht mehr „Open Educational Practices“ für den Umgang mit „Open Educational Resources“ - ein Plädoyer». Zeitschrift für Hochschulentwicklung 8(4): 56-68. http://www.zfhe.at/index.php/zfhe/ article/view/579.

Mayrberger, Kerstin, und Olaf Zawacki-Richter. 2017. «Qualität von OER - auf dem Weg zu einem deutschen Modell». In Synergie. Fachmagazin für Digitalisierung in der Lehre (4): 78 81. https://doi.org/10.25592/978.3.924330.61.3.

Mittelstrass, Jürgen. 2002. «Bildung und ethische Maße». In Die Zukunft der Bildung, hrsg. v. Nelson Killius, Jürgen Kluge, und Linda Reisch. 151-170. 4. Aufl., Suhrkamp.

Wiley, David. 2014. The Access Compromise and the 5th R. https://opencontent.org/blog/archives/3221. 